\title{
Baryonic states in supersymmetric Yang-Mills theory
}

\section{Sajid Ali*}

Institut für Theoretische Physik, Universität Münster, Wilhelm-Klemm-Str. 9, D-48149 Münster

Department of Physics, Government College University Lahore, Lahore 54000, Pakistan

E-mail: sajid.ali@uni-muenster.de

\section{Georg Bergner, Camilo Lopez}

Theoretisch-Physikalisches Institut, Universität Jena, Max-Wien-Platz 1, D-07743 Jena

E-mail: georg.bergner@uni-jena.de, camilo.lopez@uni-jena.de

\section{Henning Gerber, Gernot Münster}

Institut für Theoretische Physik, Universität Münster, Wilhelm-Klemm-Str. 9, D-48149 Münster

E-mail: h.gerber@uni-muenster.de, munsteg@uni-muenster.de

\section{Istvan Montvay}

Deutsches Elektronen-Synchrotron DESY, Notkestr. 85, D-22603 Hamburg, Germany E-mail:

montvay@mail.desy.de

\section{Stefano Piemonte}

Institute for Theoretical Physics, Universität Regensburg, Universitätsstr. 31, D-93053

Regensburg

E-mail: stefano.piemonte@ur.de

\section{Philipp Scior}

Fakultät für Physik Universität Bielefeld Universitätsstr. 25, D-33615 Bielefeld

E-mail: scior@physik.uni-bielefeld.de

In $\mathscr{N}=1$ supersymmetric Yang-Mills theory the superpartner of the gluon is the gluino, which is a spin 1/2 Majorana particle in the adjoint representation of the gauge group. Combining three gluinos, it is possible to form colour neutral bound states, analogous to baryons in QCD. The correlation functions of the corresponding baryonic operators contain a contribution represented by a "sunset diagram", and in addition, unlike in QCD, another contribution represented by a "spectacle diagram". We present first results from an implementation and calculation of these objects, obtained from numerical simulations of supersymmetric Yang-Mills theory.

The 36th Annual International Symposium on Lattice Field Theory - LATTICE2018

22-28 July, 2018

Michigan State University, East Lansing, Michigan, USA.

\footnotetext{
*Speaker.
} 


\section{Introduction}

Despite being an extremely successful effective theory, the Standard Model of particle physics needs some extensions. $\mathscr{N}=1$ supersymmetric Yang-Mills theory (SYM) is the supersymmetric extension of the gluonic sector of the Standard Model. The superpartner of the gluon is the gluino. Gauge invariance together with supersymmetry requires gluinos to transform according to the adjoint representation of the gauge group $\mathrm{SU}(N)$. SYM is similar to QCD in some aspects. It is asymptotically free at high energies, and due to confinement, the low-energy degrees of freedom are expected to be colour-neutral bound states of gluons and gluinos [1]. Their masses can be computed non-perturbatively using powerful computing machines.

Supersymmetry (SUSY) is the essential feature distinguishing SYM from QCD. If SUSY is unbroken in the continuum, as non-perturbative studies of the supersymmetric Ward identities indicate [2], the bound states are arranged in supermultiplets, see [3, 4] for numerical investigations for gauge groups $\mathrm{SU}(2)$ and $\mathrm{SU}(3)$. Effective actions for the low-energy degrees of freedom in SYM have been constructed in [5] and extended in [6,7]. They predict supermultiplets consisting of mesons, glueballs, and gluino-glue bound states. It is, however, also possible to construct colour singlet bound states of three gluinos, analogous to baryons in QCD. These baryonic states are not described by the low-energy effective actions $[5,6]$ and it is unknown how they fit into the supermultiplets. In this paper we investigate these interesting objects.

\section{2. $\mathscr{N}=1$ supersymmetric Yang-Mills theory}

The continuum Lagrangian of SYM in Euclidean space-time is given by

$$
\mathscr{L}=\frac{1}{4} F_{\mu \nu}^{a} F_{\mu \nu}^{a}+\frac{1}{2} \bar{\lambda}^{a} \gamma_{\mu}\left(\mathscr{D}_{\mu} \lambda\right)^{a}+\frac{m_{g}}{2} \bar{\lambda}^{a} \lambda^{a},
$$

where $F_{\mu \nu}^{a}$ is the non-Abelian field strength tensor built from the gauge field $A_{\mu}(x)$, which represents the gluon. The gluino field $\lambda(x)$, the fermion superpartner of the gluon, is minimally coupled to the gauge field. The gluino satisfies the Majorana condition

$$
\bar{\lambda}(x)=\lambda^{T}(x) C,
$$

where $C$ is the charge conjugation matrix. The gluino transforms under the adjoint representation of the gauge group. The covariant derivative thus acts as

$$
\left(\mathscr{D}_{\mu} \lambda\right)^{a}=\partial_{\mu} \lambda^{a}+g_{0} f_{b c}^{a} A_{\mu}^{b} \lambda^{c} .
$$

A non-zero mass $m_{g}$ of the gluino in supersymmetric Yang-Mills theory causes a soft breaking of supersymmetry. For vanishing gluino mass it is expected that supersymmetry is unbroken in the continuum [8].

For non-perturbative studies of SYM the theory is regularised on a Euclidean space-time lattice. The introduction of a space-time lattice regulator necessarily breaks supersymmetry [9]. Models that preserve part of an extended superalgebra are discussed in Ref. [10]. In our simulations, we use the "Curci-Veneziano" lattice action [11]

$$
S=S_{g}+S_{f}
$$


where the gauge part $\left(S_{g}\right)$ of the full action is given by the standard Wilson action

$$
S_{g}=\frac{\beta}{2} \sum_{x} \sum_{\mu \neq v}\left(1-\frac{1}{N_{c}} \operatorname{Retr} U_{\mu v}(x)\right) .
$$

Here $\beta \equiv 2 N_{c} / g_{0}^{2}$ is the bare lattice gauge coupling for the $\mathrm{SU}\left(N_{c}\right)$ gauge field and $U_{\mu v}(x)$ is the standard plaquette variable. The fermion part of the action in equation (2.4) is

$$
\begin{aligned}
S_{f} \equiv & \frac{1}{2} \sum_{x}\left\{\bar{\lambda}^{a}(x) \lambda^{a}(x)-\kappa \sum_{\mu=1}^{4}\left[\bar{\lambda}^{a}(x+\hat{\mu}) V_{\mu}^{a b}(x)\left(1+\gamma_{\mu}\right) \lambda^{b}(x)+\bar{\lambda}^{a}(x) V_{\mu}^{a b T}(x)\left(1-\gamma_{\mu}\right) \lambda^{b}(x+\hat{\mu})\right]\right. \\
& \left.+\frac{i}{4} g_{0} \kappa c_{S W} \bar{\lambda}^{a}(x) \sigma_{\mu v} P_{\mu v ; a b}^{(c l)}(x) \lambda^{b}(x)\right\} .
\end{aligned}
$$

Here $\kappa$ is the hopping parameter, which is related to the bare gluino mass by $\kappa=1 /\left(2 m_{g}+8\right), \gamma_{\mu}$ denotes a Dirac matrix, and $\sigma_{\mu v}=-\frac{1}{2}\left[\gamma_{\mu}, \gamma_{v}\right]$. The links $V_{\mu}^{a b}(x)$ are the gauge field variables in the adjoint representation, obtained from links $U_{\mu}(x)$ in the fundamental representation by

$$
V_{\mu}^{a b}(x) \equiv 2 \operatorname{tr}\left(U_{\mu}^{\dagger}(x) T^{a} U_{\mu}(x) T^{b}\right)
$$

where $T^{a}$ are the group generators of $\mathrm{SU}\left(N_{c}\right)$. Finally, the last term in the fermion action contains the clover-symmetrised lattice field strength tensor:

$$
P_{\mu \nu}^{(c l)}(x)=\frac{1}{4 a} \sum_{i=1}^{4} \frac{1}{2 \mathrm{i} g_{0} a}\left(U_{\mu \nu}^{(i)}(x)-U_{\mu \nu}^{(i) \dagger}(x)\right) .
$$

The coefficient $c_{S W}$ is tuned up to one-loop order in perturbation theory to improve the convergence of on-shell observables to the continuum limit [12].

\section{Baryons in Supersymmetric Yang-Mills theory}

By combining three gluino fields $\lambda(x)$, it is possible to construct a colourless object, provided all the colour indices are contracted with indices of invariant colour tensors $t_{a b c}$ for $a, b, c=$ $1, \ldots, N_{c}^{2}-1$. The corresponding particles are analogous to baryons in Quantum Chromodynamics (QCD), and will therefore also be called "baryons". Due to the fact that gluinos are in the adjoint representation of the gauge group, these objects can be constructed for both $\mathrm{SU}(2)$ and $\mathrm{SU}(3)$.

Interpolating fields for baryons will generally be of the form

$$
W(x)=t_{a b c} \lambda_{a}(x)\left(\lambda_{b}^{T}(x) \Gamma \lambda_{c}(x)\right)
$$

with an invariant colour tensor $t_{a b c}$ and a matrix $\Gamma$ in Dirac space. In the case of SU(2) one has $t_{a b c}=\varepsilon_{a b c}$ and $\Gamma$ has to be symmetric. For $\mathrm{SU}(3)$ the $t_{a b c}$ can be chosen as the completely antisymmetric structure constants $f_{a b c}$ or as the completely symmetric tensor $d_{a b c}$. The spin matrix $\Gamma$ then has to have the opposite symmetry type in order that $W \neq 0$. Depending on the choice of $\Gamma$, the baryon field $W(x)$ carries either spin $1 / 2$ only or both spin $1 / 2$ and spin $3 / 2$. These parts can be singled out with suitable projection operators [13]. A possibility, which we considered, is the choice $t_{a b c}=\varepsilon_{a b c}$ and $\Gamma=C \gamma_{4}$ for gauge group $\mathrm{SU}(2)$.

The conjugate field is

$$
\bar{W}(x)=-(C W(x))^{T} .
$$


The masses of baryons can be determined from the corresponding correlation functions

$$
\begin{aligned}
D(x, y) & =\langle W(x) \bar{W}(y)\rangle \\
D^{\alpha \delta}(x, y) & =\left\langle W^{\alpha}(x) \bar{W}^{\delta}(y)\right\rangle \\
& =-\left\langle W^{\alpha}(x) C^{\delta \alpha^{\prime}} W^{\alpha^{\prime}}(y)\right\rangle \\
& =-t_{a b c} t_{a^{\prime} b^{\prime} c^{\prime}} \Gamma^{\beta \gamma} \Gamma^{\beta^{\prime} \gamma^{\prime}} C^{\delta \alpha^{\prime}}\left\langle\lambda_{a}^{\alpha}(x) \lambda_{b}^{\beta}(x) \lambda_{c}^{\gamma}(x) \lambda_{a^{\prime}}^{\alpha^{\prime}}(y) \lambda_{b^{\prime}}^{\beta^{\prime}}(y) \lambda_{c^{\prime}}^{\gamma^{\prime}}(y)\right\rangle .
\end{aligned}
$$

The fermionic expectation value can be decomposed into gluino propagators by means of Wick's theorem. In SYM, in contrast to QCD, every pair of fermion fields contributes due to the Majorana nature of the gluino. In order to obtain the Wick contractions, it is convenient to express the correlation function in terms of six $\lambda$, by converting factors $\bar{\lambda}$ into $\lambda$ by means of the Majorana condition. The Wick contractions are

$$
\left\langle\lambda_{a}^{\alpha}(x) \lambda_{b}^{\beta}(y)\right\rangle=K_{a b}^{\alpha \beta}(x, y)=-(\triangle(x, y) C)_{a b}^{\alpha \beta},
$$

where $\triangle=Q^{-1}$ is the fermion propagator. Taking into account the fermionic signs, we get 15 terms:

$$
\begin{aligned}
D^{\alpha \alpha^{\prime}}(x, y)= & t_{a^{\prime} b^{\prime} c^{\prime}} t_{a b c} \Gamma^{\beta \gamma} \Gamma^{\beta^{\prime} \gamma^{\prime}} \times\{ \\
& +2 \triangle_{a a^{\prime}}^{\alpha \alpha^{\prime}}(x, y) \triangle_{b b^{\prime}}^{\beta \beta^{\prime}}(x, y) \triangle_{c c^{\prime}}^{\gamma \gamma^{\prime}}(x, y) \\
& +4 \triangle_{a b^{\prime}}^{\alpha \beta^{\prime}}(x, y) \triangle_{b c^{\prime}}^{\beta \gamma^{\prime}}(x, y) \triangle_{c a^{\prime}}^{\gamma \alpha^{\prime}}(x, y) \\
& +2 \triangle_{a b}^{\alpha \beta}(x, x) \triangle_{c a^{\prime}}^{\delta \alpha^{\prime}}(x, y) \triangle_{c^{\prime} b^{\prime}}^{\delta^{\prime} \beta^{\prime}}(y, y) C^{\gamma \delta} C^{\gamma^{\prime} \delta^{\prime}} \\
& +4 \triangle_{a b}^{\alpha \beta}(x, x) \triangle_{b^{\prime} c}^{\beta^{\prime} \gamma}(y, x) \triangle_{c^{\prime} a^{\prime}}^{\gamma \alpha^{\prime}}(y, y) \\
& +1 \triangle_{a a^{\prime}}^{\alpha \alpha^{\prime}}(x, y) \triangle_{b c}^{\beta \delta}(x, x) \triangle_{c^{\prime} b^{\prime}}^{\delta^{\prime} \beta^{\prime}}(y, y) C^{\gamma \delta} C^{\delta^{\prime} \gamma^{\prime}} \\
& \left.+2 \triangle_{a c}^{\alpha \delta^{\prime}}(x, y) \triangle_{b c}^{\beta \delta}(x, x) \triangle_{b^{\prime} a^{\prime}}^{\beta^{\prime} a^{\prime}}(y, y) C^{\gamma \delta} C^{\gamma^{\prime} \delta^{\prime}}\right\} .
\end{aligned}
$$

Depending on the topology of the associated fermion line diagrams, the contributions can be divided into two types called "Sunset" and "Spectacle", which are represented in Fig.1.
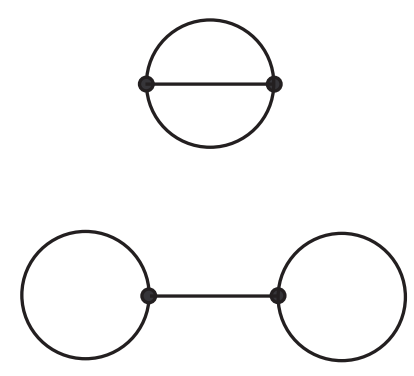

Figure 1: Contributions to the propagator of a three-gluino state. The second contribution, which we call "spectacle graph" comes from contractions of the form $\lambda(x) \lambda(x)$, which are allowed for Majorana fermions. 
The corresponding algebraic expressions are given by

$$
\begin{aligned}
& D_{\text {sunset }}(x, y)=t_{a^{\prime} b^{\prime} c^{\prime}} t_{a b c} \Gamma^{\beta \gamma} \Gamma^{\beta^{\prime} \gamma^{\prime}} P_{ \pm}^{\alpha \alpha^{\prime}} \times\{ \\
& +2 \triangle_{a a^{\prime}}^{\alpha \alpha^{\prime}}(x, y) \triangle_{b b^{\prime}}^{\beta \beta^{\prime}}(x, y) \triangle_{c c^{\prime}}^{\gamma \gamma}(x, y) \\
& \left.+4 \triangle_{a b^{\prime}}^{\alpha \beta^{\prime}}(x, y) \triangle_{b c^{\prime}}^{\beta \gamma^{\prime}}(x, y) \triangle_{c a^{\prime}}^{\gamma \alpha^{\prime}}(x, y)\right\} \\
& D_{\text {spectacle }}(x, y)=t_{a^{\prime} b^{\prime} c^{\prime}} t_{a b c} \Gamma^{\beta \gamma} \Gamma^{\beta^{\prime} \gamma^{\prime}} P_{ \pm}^{\alpha \alpha^{\prime}} \times\{ \\
& +2 \triangle_{a b}^{\alpha \beta}(x, x) \triangle_{c a^{\prime}}^{\delta \alpha^{\prime}}(x, y) \triangle_{c^{\prime} b^{\prime}}^{\delta^{\prime} \beta^{\prime}}(y, y) C^{\gamma \delta} C^{\gamma^{\prime} \delta^{\prime}} \\
& +4 \triangle_{a b}^{\alpha \beta}(x, x) \triangle_{b^{\prime} c}^{\beta^{\prime} \gamma}(y, x) \triangle_{c^{\prime} a^{\prime}}^{\gamma^{\prime} \alpha^{\prime}}(y, y) \\
& +1 \triangle_{a a^{\prime}}^{\alpha \alpha^{\prime}}(x, y) \triangle_{b c}^{\beta \delta}(x, x) \triangle_{c^{\prime} b^{\prime}}^{\delta^{\prime} \beta^{\prime}}(y, y) C^{\gamma \delta} C^{\delta^{\prime} \gamma^{\prime}} \\
& \left.+2 \triangle_{a c}^{\alpha \delta^{\prime}}(x, y) \triangle_{b c}^{\beta \delta}(x, x) \triangle_{b^{\prime} a^{\prime}}^{\beta^{\prime} \alpha^{\prime}}(y, y) C^{\gamma \delta} C^{\gamma^{\prime} \delta^{\prime}}\right\}
\end{aligned}
$$

In contrast to mesonic or glueball correlation functions, the baryonic correlation functions are not symmetric or antisymmetric with respect to Euclidean time, if no parity projection is included, see e.g. [14]. Therefore we include the projection onto parity eigenstates by means of the parity projector $P_{ \pm}$, which is defined as $P_{ \pm}=\frac{1}{2}\left(1 \pm \gamma_{4}\right)$ for zero momentum.

We have implemented the contributions to the baryonic correlation function into the measurement code of our SYM collaboration. The numerically quite expensive part is the evaluation of the spectacle piece. We have calculated it by means of combining the exact contribution of the lowest eigenmodes of the Wilson-Dirac matrix with the stochastic estimator technique.

Presently some preliminary results have been obtained. Fig. 2 shows the two pieces of the baryonic correlation function and its sum for gauge group $\mathrm{SU}(2)$. In this case the sunset piece

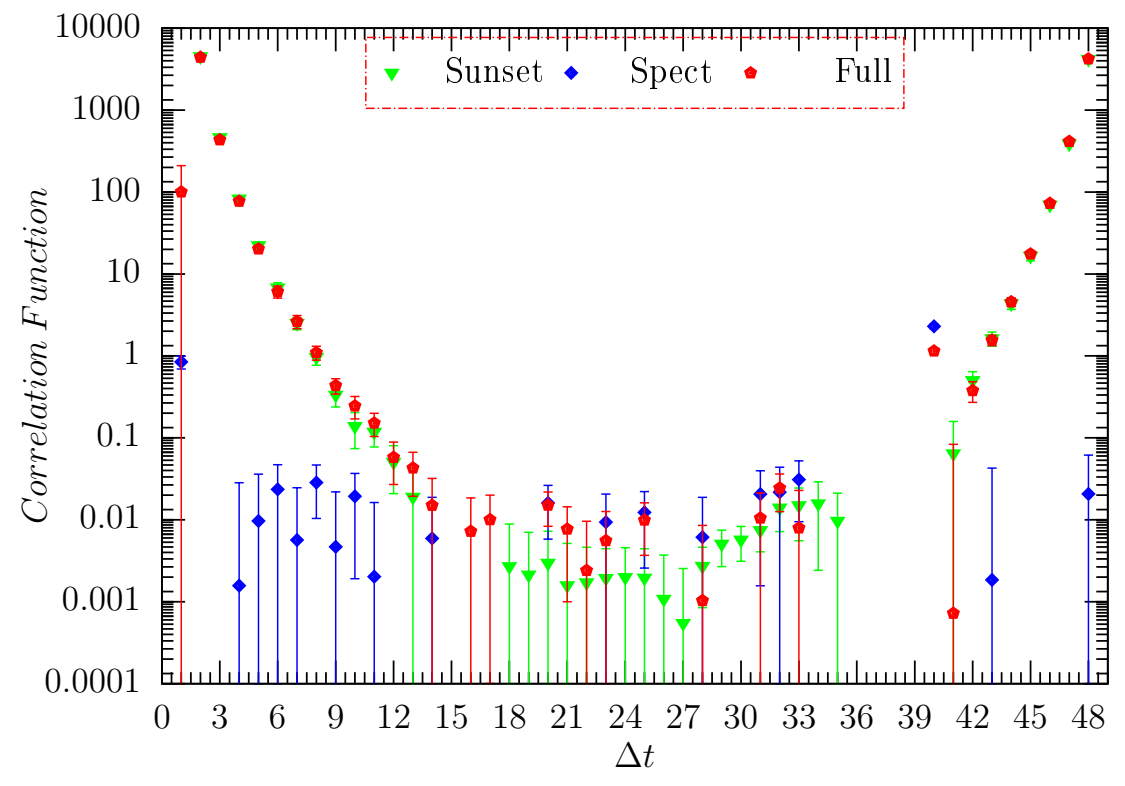

Figure 2: Numerical results for correlation functions at each time slice for gauge group SU(2), lattice volume, $V=24^{3} \cdot 48, \beta=1.75$ and $\kappa=0.14925$. 

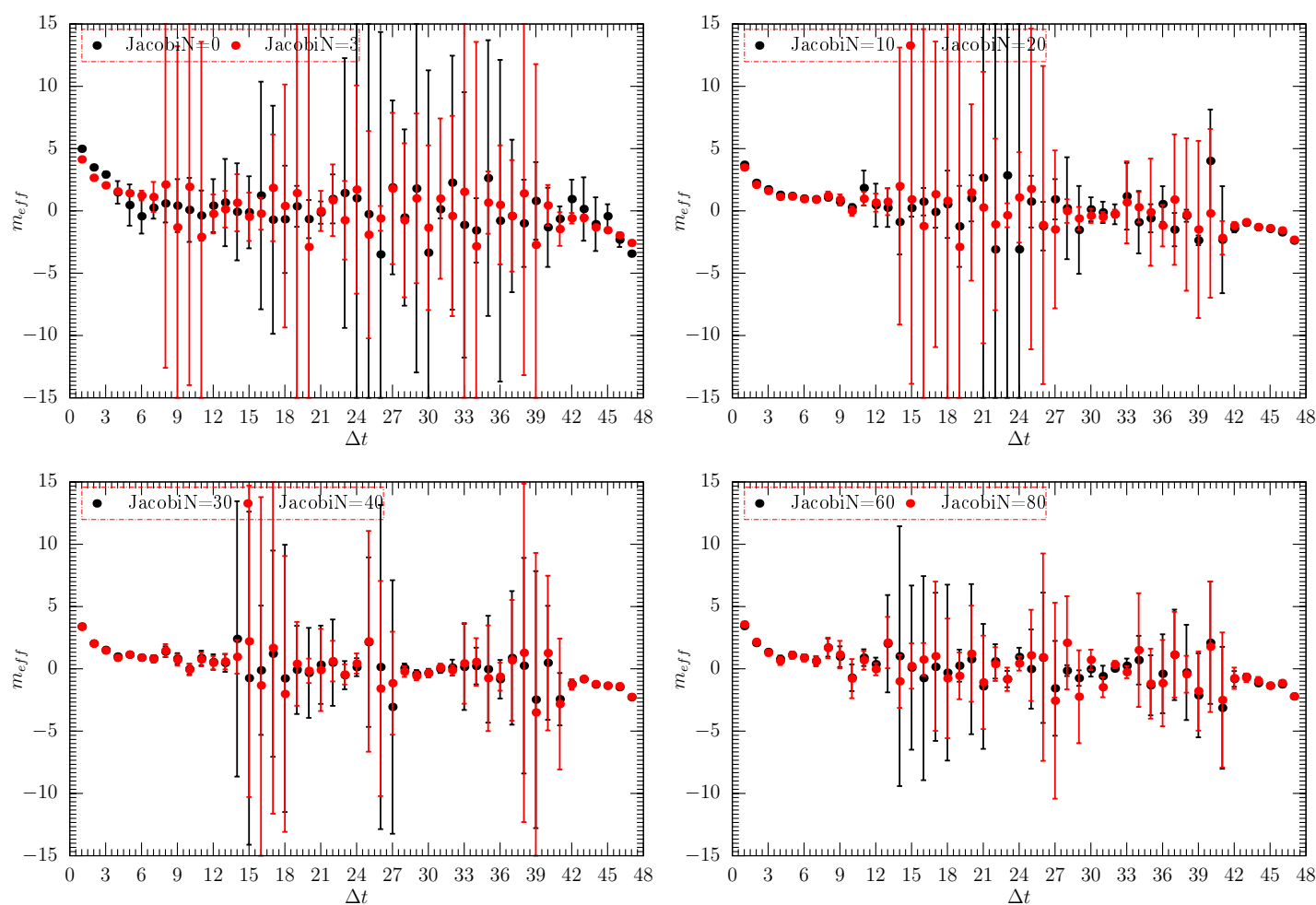

Figure 3: Effective mass plots for fixed APE pre-smearing with level 40 and different levels of Jacobi smearing of the Dirac fermion matrix.

appears to dominate the correlation function for not too large times. Fig. 3 shows the effective mass for different smearings.

\section{Conclusion and outlook}

In this paper we constructed the correlation function for a three-gluino state in supersymmetric Yang-Mills theory and presented preliminary estimates of its mass using Monte Carlo simulation at non-zero gluino mass and at finite inverse gauge coupling $\beta$. With better numerical results we expect to be able to perform chiral and continuum extrapolations in the future, and to find out how the baryonic states fit into the supermultiplet found in Ref. [3].

\section{Acknowledgements}

The authors gratefully acknowledge the Gauss Centre for Supercomputing e. V. (www.gausscentre.eu) for funding this project by providing computing time on the GCS Supercomputer JUQUEEN and JURECA at Jülich Supercomputing Centre (JSC) and SuperMUC at Leibniz Supercomputing Centre (LRZ). Further computing time has been provided on the compute cluster PALMA of the University of Münster. This work is supported by the Deutsche Forschungsgemeinschaft (DFG) through the Research Training Group "GRK 2149: Strong and Weak Interactions-from Hadrons to Dark Matter". G. Bergner and C. Lopez acknowledge support from the Deutsche Forschungsge- 
meinschaft (DFG) under Grant No. BE 5942/2-1. S. Ali acknowledges financial support from the Deutsche Akademische Austauschdienst (DAAD).

\section{References}

[1] D. Amati, K. Konishi, Y. Meurice, G. C. Rossi and G. Veneziano, Nonperturbative aspects in supersymmetric gauge theories, Phys. Rept. 162 (1988) 169.

[2] S. Ali, G. Bergner, H. Gerber, P. Giudice, I. Montvay, G. Münster, S. Piemonte and P. Scior, Analysis of Ward identities in supersymmetric Yang-Mills theory, Eur. Phys. J C 78 (2018) 404 [arXiv: 1802.07067 [hep-lat]].

[3] G. Bergner, P. Giudice, I. Montvay, G. Münster and S. Piemonte, The light bound states of supersymmetric SU(2) Yang-Mills theory, JHEP 1603 (2016) 080 [arXiv: 1512.07014 [hep-lat]].

[4] S. Ali, G. Bergner, H. Gerber, P. Giudice, I. Montvay, G. Münster, S. Piemonte and P. Scior, The light bound states of $\mathscr{N}=1$ supersymmetric SU(3) Yang-Mills theory on the lattice, JHEP 1803 (2018) 113 [arXiv: 1801.08062 [hep-lat]].

[5] G. Veneziano and S. Yankielowicz, An effective Lagrangian for the pure $\mathscr{N}=1$ supersymmetric Yang-Mills theory, Phys. Lett. B 113 (1982) 231.

[6] G. R. Farrar, G. Gabadadze and M. Schwetz, On the effective action of $N=1$ supersymmetric Yang-Mills theory, Phys. Rev. D 58 (1998) 015009 [arXiv: hep-th/9711166].

[7] G. R. Farrar, G. Gabadadze and M. Schwetz, The spectrum of softly broken $N=1$ supersymmetric Yang-Mills theory, Phys. Rev. D 60 (1999) 035002 [arXiv: hep-th/ 9806204 ].

[8] E. Witten, Constraints on Supersymmetry Breaking, Nucl. Phys. B 202 (1982) 253.

[9] G. Bergner, Complete supersymmetry on the lattice and a No-Go theorem, JHEP 1001 (2010) 024 [arXiv: 0909.4791 [hep-lat]].

[10] S. Catterall, D. B. Kaplan and M. Ünsal, Exact lattice supersymmetry, Phys. Rept. 484 (2009) 71 [arXiv: 0903.4881 [hep-lat]].

[11] G. Curci and G. Veneziano, Supersymmetry and the lattice: a reconciliation?, Nucl. Phys. B 292 (1987) 555 .

[12] S. Musberg, G. Münster and S. Piemonte, Perturbative calculation of the clover term for Wilson fermions in any representation of the gauge group $S U(N)$, JHEP 1305 (2013) 143 [arXiv: 1304.5741 [hep-lat]].

[13] D. B. Leinweber, W. Melnitchouk, D. G. Richards, A. G. Williams and J. M. Zanotti, Baryon spectroscopy in lattice QCD, Lect. Notes Phys. 663 (2005) 71, Springer, Berlin [arXiv: nucl-th/0406032].

[14] I. Montvay and G. Münster, Quantum Fields on a Lattice, Cambridge University Press, 1994, Chap. 5.2. 